\title{
Correction to: ALDH2 protects against high fat diet-induced obesity cardiomyopathy and defective autophagy: role of CaM kinase II, histone H3K9 methyltransferase SUV39H, Sirt1, and PGC-1a deacetylation
}

Shuyi Wang $\cdot$ Cong Wang $\cdot$ Subat Turdi $\cdot$ Kacy L. Richmond · Yingmei Zhang $\cdot$ Jun Ren

Published online: 27 November 2019

(c) Macmillan Publishers Limited, part of Springer Nature 2019

\section{Correction to: International Journal of Obesity} https://doi.org/10.1038/s41366-018-0030-4

The authors found a critical mistake in the assembly of Fig. 2; in Fig. 2A the right two images were erroneously duplicated. The authors have re-analysed all the data, checked for accuracy and provided the updated Fig. 2 here. Nothing is affected with regards to data summary and conclusion. 
A.
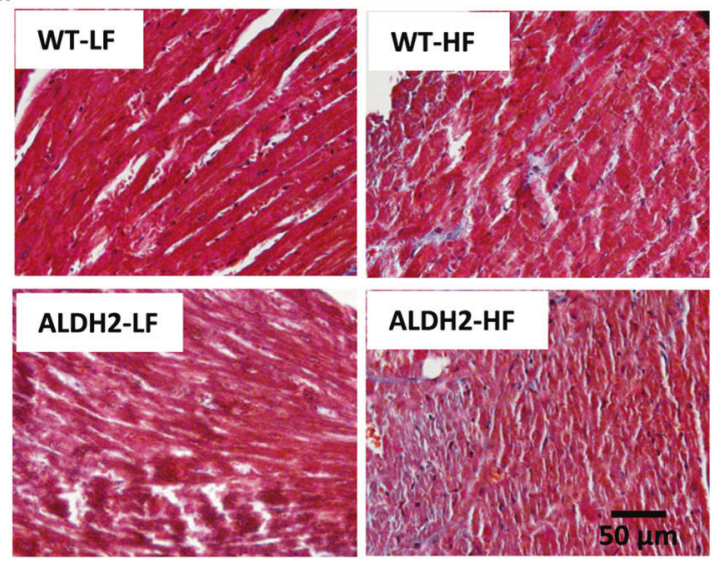

C.
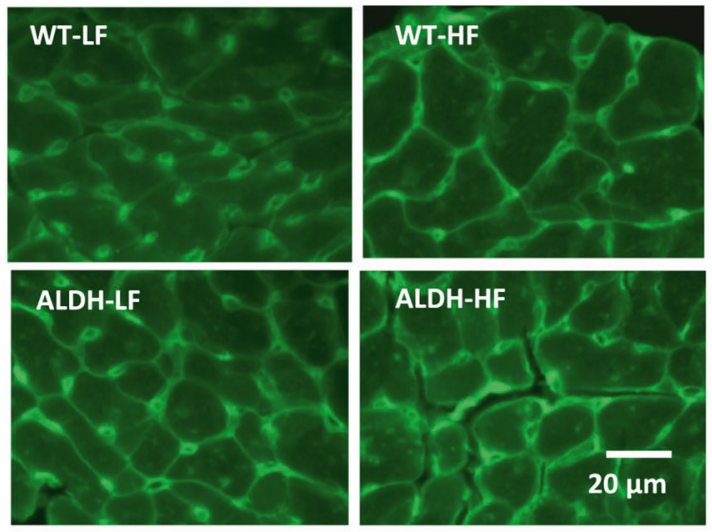

B.

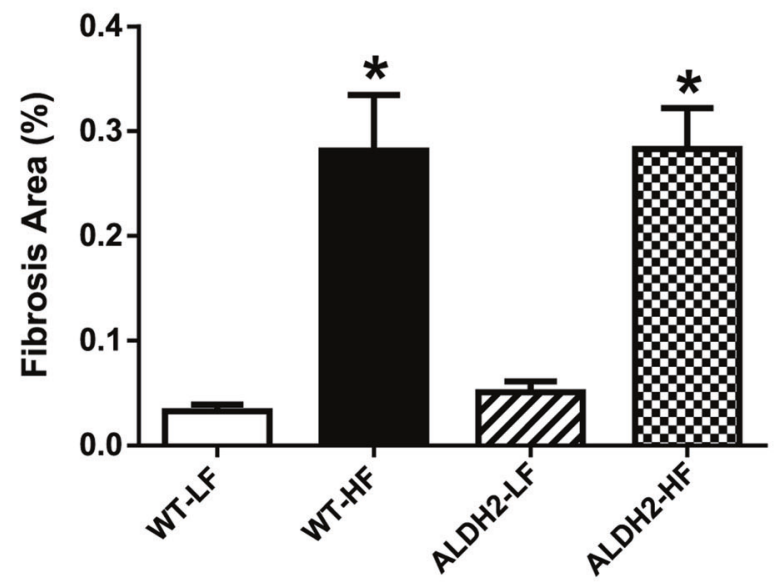

D.

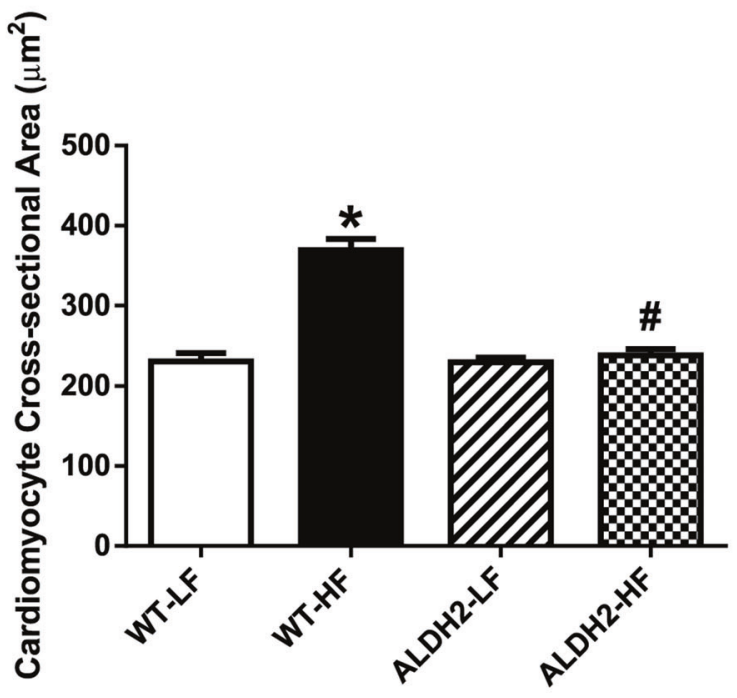

Fig. 2 Cardiac fibrosis, hypertrophy, intracellular $\mathrm{Ca}^{2+}$ properties, andsuperoxide levels in cardiomyocytes from WT and ALDH2 mice fedlow (LF) or high fat (HF) diet for 5 months. a Representative photographs of Masson trichrome staining of WT and ALDH2 mice fed LFor HF diet. b Quantitative analysis of interstitial fibrosis using measurements of $\sim 100$ cardiomyocytes from five mice per group. c Representative photographs of Lectin staining of transverse sections of left ventricular myocardium $(\times 400)$ from WT and ALDH2 mice fed
G.
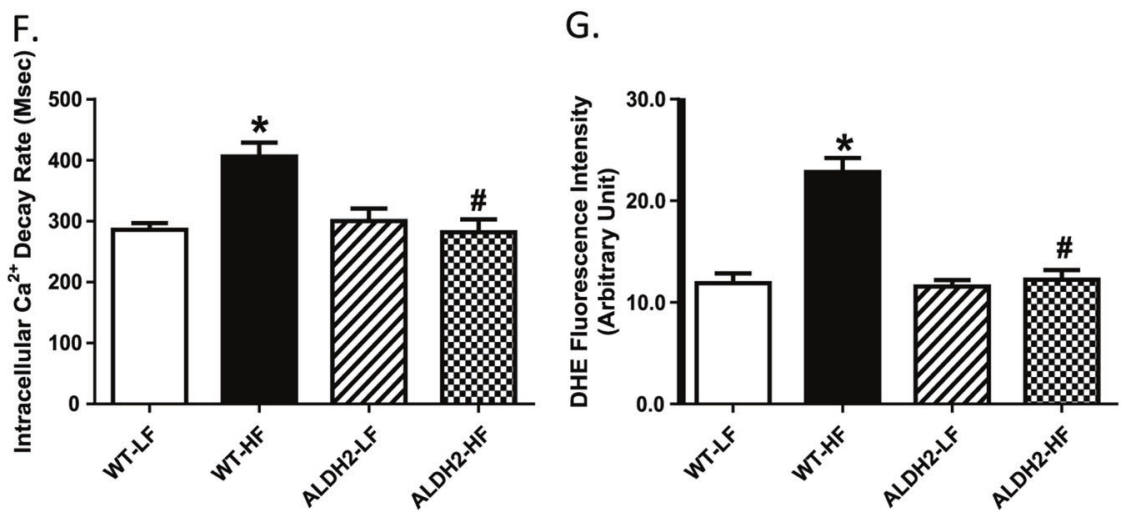

LF or HF diet. d Quantitative analysis of cardiomyocyte crosssectional (transverse) area using measurements of $\sim 100$ cardiomyocytes from five mice per group. e Electrically stimulated rise in FFI $(\Delta F F)$. $f$ Intracellular $\mathrm{Ca}^{2+}$ decay rate; and $\mathrm{g}$ superoxide levels using DHE fluorescence intensity. Mean $\pm \mathrm{SEM}, n=8-11$ mice per groupfor Masson trichrome staining; $n=12-16$ mice for lectin staining and $n=$ $60-75$ cells from three mice per group for fura-2. * $p$ WT-LF group, \#p $<0.05$ vs. WT-HF group 